\title{
Preface
}

\section{Anesthesia Outside the Operating Room}

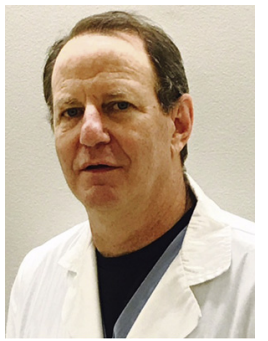

Mark S. Weiss, MD

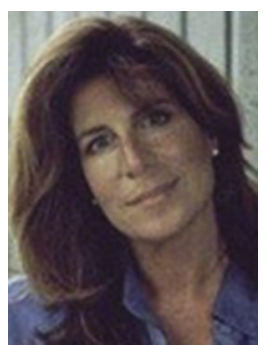

Wendy L. Gross, MD, MHCM Editors

Shortly after I arrived at the University of Pennsylvania in 2011, I attended an early morning meeting of the Non-Operating Room Anesthesia (NORA) team. I had been in private practice for years, and only a few months earlier left that practice for Penn. It was as a private practice anesthesiologist that we first noticed these "Outof-OR" cases. At first, there were only a few cases. However, over time, we were being asked to provide more services to outside areas that evoked discomfort on several levels. Among the issues that arose were: What is best for the NORA patient? How should we do these cases? What does the proceduralist need? How do we staff these services? Who pays for our time, expenses, monitors, and auxiliary staff? And when I came to Penn, I noticed rather quickly that these very same questions were being asked.

During that meeting of our Penn NORA team, we identified the upcoming surge of growth in NORA case volume. At that time, at Hospital of the University of Pennsylvania, about $6 \%$ of all our anesthesia cases occurred outside the OR. We predicted that in just a few years, more than $25 \%$ of our cases would involve NORA. (Today, we are actually doing over a third of cases in this manner.) I looked around the room and all I saw was the "Old Guard" of anesthesiologists. Being new to the department, I raised my hand and asked, "How are we training our residents for the future?" My colleagues' response was the following: "You know, we all learned how to do this on the fly..."

That is just not good enough.

Back in 2011, there were no defined "best practices" published. There was precious little direction for NORA cases nationally. The American Society of Anesthesiologists had published some recommendations, but no clear guidelines addressing what was required to care safely for patients in the NORA setting. We needed standards, guidelines, and teaching programs. In addition, economic issues needed to be 
addressed. Finally, ground rules for collaboration needed to be established to deal with the anticipated continued rise in NORA case volume.

To prepare for the future, it is helpful to examine the reasons that led to how you arrived at the present. There are several large "tectonic plates" acting as driving forces behind the groundswell of growth in NORA: an aging population with multiple medical comorbidities; the service expectations of both health care consumers and providers; cost and value for insurance companies, health care institutions, and government; and advances in various procedural techniques have resulted in continued improvements and expansion in NORA. Today, anesthesia teams are coordinating and collaborating with proceduralists from various specialties to develop standards and practices to ensure patient safety as well as the success of procedures. Anesthesiologists are involved in all aspects of the care of NORA patients throughout the hospital in multiple clinical settings.

This issue of the Anesthesiology Clinics is only a snapshot of NORA in time. Although our field has made progress, I believe that the evolution of NORA in the coming years will revolutionize the way medicine is delivered in this country, and that we will look back at this issue in several years and be pleasantly surprised at how this field has evolved. It seems as if almost every day a new procedure is demonstrated or a new technique is developed. The opportunity for improved and efficient health care is changing rapidly thanks to the innovative, collaborative, imaginative minds and efforts of those involved.

The issues and topics covered in this issue are as varied as NORA itself. When we discussed how to organize this effort, Wendy Gross and I agreed that when possible the articles in this project, just as in the NORA suite, would be a collaborative effort by an anesthesiologist and a proceduralist expert in their respective field. The results of these collaborations were a fascinating series of articles focusing on different and important aspects of NORA. This issue includes thoughtful discussions on the changing landscape of NORA care, organization, and information systems. In addition, there are interesting reviews of safety in the NORA suite using closed claims data and monitoring. Clinically, a variety of settings and procedures are examined. One procedure in particular, tumor ablation in the radiology suite, is closely examined from the view of both the anesthesia team and the radiologist, allowing us to look at our field from our colleagues' perspective. Finally, in recognition of the economic implications of the growth of NORA, we have included a section regarding market evaluation on finances, bundled payments, and Accountable Care Organizations, as well as how to plan and strategize priorities for the future.

Wendy and I would like to thank the many contributors among multiple institutions from many specialties who have contributed to the success of this project. We are thankful to Dr Lee Fleisher, Professor and Chairman of Anesthesiology at the University of Pennsylvania, for giving us the opportunity to edit this issue of the Anesthesiology Clinics, and to the professionals at Elsevier for their encouragement and 
advice with this endeavor. We are especially grateful to our families for their patience and understanding of the commitment of time that this project took to complete.

Mark S. Weiss, MD Department of Anesthesiology and Critical Care Hospital of the University of Pennsylvania

3400 Spruce Street 6th Floor Dulles Building Philadelphia, PA 19104, USA

Wendy L. Gross, MD, MHCM Division of Cardiac Anesthesia Department of Anesthesiology, Perioperative and Pain Medicine

Brigham and Women's Hospital 75 Francis Street Boston, MA 02115, USA

E-mail addresses: weissm@uphs.upenn.edu; mweiss@sonoria.org (M.S. Weiss) wgross@partners.org (W.L. Gross) 